\title{
Clinicopathologic Features of Metastasis in Nonsentinel Lymph Nodes of Breast Carcinoma Patients
}

\section{A Metaanalysis}

\author{
Amy C. Degnim, M.D. ${ }^{1}$ \\ Kent A. Griffith, M.P.H., M.s. ${ }^{2}$ \\ Michael S. Sabel, M.D. ${ }^{1}$ \\ Daniel F. Hayes, M.D. ${ }^{3}$ \\ Vincent M. Cimmino, M.D. ${ }^{1}$ \\ Kathleen M. Diehl, M.D. ${ }^{1}$ \\ Peter C. Lucas, M.D. ${ }^{4}$ \\ Matthew L. Snyder, M.D. ${ }^{4}$ \\ Alfred E. Chang, M.D. ${ }^{1}$ \\ Lisa A. Newman, M.D., M.P.H. ${ }^{1}$ \\ ${ }^{1}$ Department of Surgery, University of Michigan, \\ Ann Arbor, Michigan. \\ ${ }^{2}$ Department of Biostatistics, University of Michi- \\ gan, Ann Arbor, Michigan. \\ ${ }^{3}$ Department of Medicine, University of Michigan, \\ Ann Arbor, Michigan. \\ ${ }^{4}$ Department of Pathology, University of Michigan, \\ Ann Arbor, Michigan.
}

Supported by the Fashion Footwear Association of New York (FFANY)/QVC presents/Shoes on Sale ${ }^{\circledR}$ (to Daniel F. Hayes).

The authors wish to thank Kathryn Clark for her expert assistance with data review and article preparation.

Address for reprints: Amy C. Degnim, M.D., Department of Surgery, Mayo Clinic, 200 First Street SW, Rochester, MN 55905; Fax: (507) 284-5196; E-mail: Degnim.Amy@mayo.edu

Received June 11, 2003; revision received August 20, 2003; accepted August 22, 2003.
BACKGROUND. In breast carcinoma patients with a positive sentinel lymph node (SN), the value of complete axillary lymph node dissection has been questioned. Multiple published reports have attempted to identify clinicopathologic characteristics of the primary tumor and SN that are associated with an increased likelihood of positive nonsentinel lymph nodes (NSN). Because of differences in lymph node evaluation techniques and limited patient numbers in each study, the authors performed a meta-analysis to assess the regularity and relative strength of association between various characteristics and the risk of NSN metastasis.

METHODS. A MEDLINE search identified 15 candidate studies, 11 of which met the criteria for analysis. General elements of the studies, the pathologic characteristics evaluated, and the results for selected characteristics were compared. Original data were abstracted from each study and used to calculate odds ratios. The MantelHaenszel common odds ratios were calculated to determine the relative strength of the associations.

RESULTS. Despite methodologic differences, the correlation between positive NSNs and certain pathologic characteristics was found to be remarkably similar among studies. The 5 individual characteristics found to be associated with the highest likelihood of NSN metastasis are SN metastasis $>2 \mathrm{~mm}$ in size, extranodal extension in the SN, tumor size $>2 \mathrm{~cm},>1$ positive $\mathrm{SN}$, and lymphovascular invasion in the primary tumor.

CONCLUSIONS. There is general concordance among studies regarding the association between pathologic characteristics and NSN metastasis in breast carcinoma patients with a positive SN. The pooled analysis identified those factors with the strongest associations that should be evaluated routinely in SN specimens and included in prospective databases for the development of a predictive model. Cancer 2003;98:2307-15. (c) 2003 American Cancer Society.

KEYWORDS: nonsentinel lymph node (NSN), sentinel lymph node (SN) biopsy, breast neoplasms, predictive factors, axillary lymph node dissection (ALND), metastasis.

B ecause of the acknowledged morbidity of axillary lymph node dissection (ALND), ${ }^{1,2}$ sentinel lymph node (SN) biopsy has rapidly emerged as the primary approach to staging the axillary lymph nodes in patients with breast carcinoma. If the $\mathrm{SN}$ is positive, the standard of care remains a completion ALND, but this has been questioned because the survival benefit remains uncertain. ${ }^{3,4}$ Completion ALND may also offer an advantage in terms of reduced regional recurrence in patients who harbor residual axillary lymph node metastasis, but many patients with positive SNs have no other positive axillary lymph 
nodes. There is general agreement that removal of negative lymph nodes does not provide any significant benefit. Therefore, the ability to predict the status of the nonsentinel lymph nodes (NSNs), whether positive or negative, could help to tailor surgical therapy to those individuals most likely to derive a benefit from completion ALND. Patients with a predicted small chance of residual axillary lymph node metastasis after a positive SN biopsy might be able to safely avoid completion ALND. Conversely, prediction of the high likelihood of residual axillary lymph node metastases may identify patients for whom completion ALND is necessary.

In an attempt to obtain predictive parameters, multiple published studies have identified pathologic characteristics of the primary tumor and SN that are associated with an increased likelihood of additional positive NSNs. Because of perceived regional and institutional differences in methods of obtaining and evaluating lymph nodes, as well as limited patient numbers in each study, we undertook a comparative meta-analysis of these studies to assess the strength of the associations of these characteristics with the presence of NSN metastasis and the consistency of these findings across studies.

\section{MATERIALS AND METHODS}

To identify relevant articles, a MEDLINE search was performed using the following keywords: nonsentinel node, non-sentinel node, nonsentinel lymph node, non-sentinel lymph node, sentinel lymph node biopsy combined with breast neoplasms and characteristics, sentinel lymph node biopsy combined with breast neoplasms and predictors, and breast neoplasms combined with axillary dissection and metastasis. Articles from these searches with titles pertinent to the topic were evaluated further. Articles included in the meta-analysis were those that reported original data regarding the association between various patient/ tumor characteristics and the likelihood of positive NSNs. Criteria for inclusion in the meta-analysis were as follows: 1) the study identified the population of patients with a positive SN and who underwent completion ALND, 2) original data were reported on the number of SN-positive patients who had positive NSNs stratified by various patient/tumor characteristics, 3) statistical analysis for these characteristics was not based on groups that included patients with negative SNs, and 4) the study population included patients with both micrometastatic and macrometastatic disease in the SNs. References cited in the selected articles were reviewed for additional relevant reports, and these studies were subjected to the same criteria for inclusion. Studies that appeared to represent over- lapping patient populations were evaluated for extent of data; the study with lesser relevance was excluded.

Information was collected concerning the characteristics of the studies, as well as the types of patient/ tumor characteristics examined and the level of statistical significance. The numbers of patients with positive and negative NSNs for each characteristic were abstracted from each study. In some studies, data were not presented for characteristics that were not statistically significant. Therefore, a complete dataset was not always obtainable for every characteristic evaluated in each study. The accuracy of the abstracted data was verified independently by an external consultant (ion Write, Saline, MI). These data then were used to construct graphical representations of the association between the selected characteristics and positive NSNs (as determined by hematoxylin and eosin $[\mathrm{H} \& \mathrm{E}]$ evaluation unless otherwise specified). Study-specific odds ratios were calculated from the abstracted data, and the common odds ratios across studies were estimated using the Mantel-Haenszel method. ${ }^{5}$ For the meta-analysis, data regarding NSNs found to be positive by $\mathrm{H} \& \mathrm{E}$ evaluation were used whenever available, but data from studies in which immunohistochemistry (IHC) was performed on NSNs were included. Point estimates and 95\% confidence intervals $(95 \% \mathrm{CI})$ for the common odds ratios were reported for characteristics supported by abstracted data from at least 2 studies. The standard criterion of $P$ values $<0.05$ for hypothesis tests was used to determine statistical significance for original calculations herein and when considering hypothesis tests conducted by the individual study.

\section{RESULTS}

Based on the MEDLINE search, 15 candidate studies were identified. ${ }^{3,6-19}$ One study ${ }^{3}$ was excluded because of presumed overlap of the dataset with another study. ${ }^{10}$ Two studies ${ }^{17,18}$ were excluded because the evaluations were limited to patients with micrometastatic disease in the $\mathrm{SN}$, a group that was potentially dissimilar from the populations of the remaining studies. A fourth study ${ }^{19}$ was excluded because the data regarding completion ALND findings were correlated with features among the collective group of SN cases; data concerning the subset of SN-positive patients were not reported.

The 11 studies included in the meta-analysis were published between 1999 and 2003. The study sample sizes (patients with a positive $\mathrm{SN}$ who underwent completion ALND) ranged from 60-389 patients. Patient age, the number of SNs obtained, and the number of NSNs obtained appeared similar across studies (Table 1), although no explicit statistical testing of this 
TABLE 1

Comparison of Study Characteristics

\begin{tabular}{|c|c|c|c|c|c|c|c|c|c|}
\hline Study & $\begin{array}{l}\text { Year } \\
\text { published }\end{array}$ & $\begin{array}{l}\text { No. of } \\
\text { patients }\end{array}$ & $\begin{array}{l}\text { Age of } \\
\text { patients, } \\
\text { (yrs) } \\
\text { (median) }\end{array}$ & $\begin{array}{l}\% \text { of patients } \\
\text { with tumors } \\
\leq 2 \mathrm{~cm}\end{array}$ & $\begin{array}{l}\text { Mapping } \\
\text { technique }\end{array}$ & $\begin{array}{l}\text { IHC performed on } \\
\text { NSNs routinely if } \mathrm{H} \\
\text { \& E negative }\end{array}$ & $\begin{array}{l}\% \text { of patients } \\
\text { with } \\
\text { micrometastasis } \\
\text { in } \mathrm{SN} \text { ( } \leq 2 \mathrm{~mm})\end{array}$ & $\begin{array}{l}\text { No. of SNs } \\
\text { obtained, } \\
\text { (median) }\end{array}$ & $\begin{array}{l}\text { No. of } \\
\text { NSNs } \\
\text { obtained, } \\
\text { (median) }\end{array}$ \\
\hline Abdessalam et al. ${ }^{6}$ & 2001 & 100 & $54(30-83)$ & 49 & Dye + iso & No & 35 & $2.4 \pm 2.2^{\mathrm{b}}$ & $22.1 \pm 8.6^{\mathrm{b}}$ \\
\hline Weiser et al. ${ }^{7}$ & 2001 & 206 & $52(21-85)$ & 74 & Dye + iso & No & 46 & NR & NR \\
\hline Rahusen et al. ${ }^{8}$ & 2001 & 93 & NR & 57 & Dye + iso & Yes & $\mathrm{NA}$ & $1.7(1-4)^{\mathrm{b}}$ & NR \\
\hline Wong et al. ${ }^{9}$ & 2001 & 389 & NR & 50 & $\begin{array}{l}\text { Dye, iso, } \\
\text { or both }\end{array}$ & No & NR & $2.2^{\mathrm{b}, \mathrm{c}}$ & $13^{\mathrm{b}, \mathrm{c}}$ \\
\hline Turner et al. ${ }^{10}$ & 2000 & 194 & $52(28-91)$ & 54 & Dye \pm iso & Yes & 48 & $2 \quad(1-7)$ & $17(7-59)$ \\
\hline Hwang et al. ${ }^{11}$ & 2003 & 131 & $53(30-78)$ & 55 & Dye \pm iso & No & 25 & $2 \quad(1-8)$ & $15(3-37)$ \\
\hline Reynolds et al. ${ }^{12}$ & 1999 & 60 & $56(26-90)$ & 60 & Dye \pm iso & $\begin{array}{l}\text { Yes, for T1 with } \\
\text { micrometastasis }\end{array}$ & 45 & NR & NR \\
\hline Cserni $^{13}$ & 2001 & 69 & NR & 30 & Dye & Unknown & NA & $1.4(1-6)^{\mathrm{b}, \mathrm{c}}$ & $17(5-42)^{\mathrm{b}, \mathrm{c}}$ \\
\hline Kamath et al. ${ }^{14}$ & 2001 & 101 & NR & NR & Dye + iso & Unknown & 46 & NR & NR \\
\hline Mignotte et al. ${ }^{15}$ & 2002 & 120 & NR & 78 & Dye \pm iso & No & 57 & $1.8(1-6)^{\mathrm{b}, \mathrm{c}}$ & NR \\
\hline Canavese et al. ${ }^{16}$ & 2001 & 72 & $61(40-79)^{b}$ & 68 & Dye \pm iso & Unknown & 29 & $1.4(1-4)^{\mathrm{b}, \mathrm{c}}$ & NR \\
\hline
\end{tabular}

IHC: immunohistochemistry; NSNs: nonsentinel lymph nodes; H \& E: hematoxylin and eosin; SN: sentinel lymph nodes; isotope: ; NR: data not reported; NA: not applicable.

${ }^{a}$ Number of patients in the study who had a positive sentinel lymph node biopsy and completion axillary lymph node dissection.

${ }^{\mathrm{b}}$ Reported as the mean (range) or mean \pm standard deviation.

${ }^{\mathrm{c}}$ Calculated mean includes some patients with nonsentinel lymph nodes.

hypothesis was possible given the format of the data. In contrast, the study populations exhibited some apparent variation in disease stage distribution; the proportions of patients with tumors $\leq 2 \mathrm{~cm}$ and with SN micrometastasis ranged from $30-78 \%$ and $25-57 \%$, respectively.

The routine use of IHC and additional sections to evaluate the NSNs may be the most important difference among the studies. The SN hypothesis was validated by confirming that IHC and additional sectioning rarely detect NSN metastasis in patients with SNs found to be negative by $\mathrm{H} \& \mathrm{E}$ staining and $\mathrm{IHC}^{20,21}$ However, in patients with SNs found to be positive by $\mathrm{H} \& \mathrm{E}$ staining or IHC, increased scrutiny of NSNs with IHC and additional sections reportedly results in a $10-20 \%$ increase in the detection of NSN metastasis. $^{21,22}$ Of the studies analyzed, five used only $\mathrm{H} \& \mathrm{E}$ staining for the evaluation of NSNs. ${ }^{6,7,9,11,15}$ In addition to $\mathrm{H} \& \mathrm{E}$ staining, three studies performed IHC and additional sections on NSNs. Of these 3 studies, 1 evaluated NSNs on 2 levels with a $250-\mu \mathrm{m}$ interval, ${ }^{8} 1$ evaluated NSNs on 2 levels with a $40-\mu \mathrm{m}$ interval, ${ }^{10}$ and 1 evaluated 3-8 levels in the subgroup of patients with primary tumors measuring $<2 \mathrm{~cm}$ and micrometastasis in the SN. ${ }^{12}$ In the remaining three studies, the histologic evaluation of the NSNs was not able to be determined. ${ }^{13,14,16}$ One study reported data concerning NSNs both with and without the use of IHC for three characteristics: tumor size, metastasis size, and lymphovascular invasion (LVI). ${ }^{10}$ Data regarding the remaining factors reported in that study reflect the use of IHC on NSNs. We constructed graphs based on data reporting NSNs found to be positive by $\mathrm{H} \&$ E evaluation unless otherwise specified. As stated in the "Methods" section, the meta-analysis utilized data regarding NSNs found to be positive by $\mathrm{H} \& \mathrm{E}$ evaluation whenever available, but data from studies performing IHC on NSNs also were included in the meta-analysis.

Table 2 presents the clinicopathologic characteristics that were evaluated by at least two studies. Whether the characteristic was found to have a statistically significant association with the presence of NSN metastasis is reported. Two studies reported apparent differences in the proportion of NSN metastasis based on tumor size and metastasis size but did not perform a statistical test of that hypothesis. ${ }^{15,16}$ The characteristics found to be statistically significant by three or more studies were: tumor size, size of metastatic focus within the SN, presence of extranodal extension (EE), presence of LVI, and the number of positive SNs. Other characteristics that were found to be associated significantly with NSN metastasis by univariate analysis in only one study included higher histologic grade, negative estrogen receptor status, and three or more SNs removed.

In addition to the characteristics shown in Table 2 , three additional factors were found to be significantly associated with the presence of NSN metastasis, each in a separate study. In one study that evaluated the location of metastasis in the $\mathrm{SN}^{13}$ a metastasis 
TABLE 2

Association between Clinicopathologic Features and NSN Metastasis by Study

\begin{tabular}{|c|c|c|c|c|c|c|c|c|c|c|c|c|c|c|}
\hline Study & $\begin{array}{l}\text { Tumor } \\
\text { size }\end{array}$ & $\begin{array}{l}\text { Metastasis } \\
\text { size }\end{array}$ & $\begin{array}{l}\text { Extranodal } \\
\text { extension }\end{array}$ & LVI & $\begin{array}{l}\text { Histologic } \\
\text { grade }\end{array}$ & Age & ER & PR & $\begin{array}{l}\text { Histologic } \\
\text { type }\end{array}$ & $\begin{array}{l}\text { No. of } \\
\text { positive } \\
\text { SNs }\end{array}$ & $\begin{array}{l}\text { Total no. } \\
\text { of SNs }\end{array}$ & $\begin{array}{l}\text { HER-2- } \\
\text { neu }\end{array}$ & $\begin{array}{l}\text { Tumor } \\
\text { palpable }\end{array}$ & $\begin{array}{l}\text { Tumor } \\
\text { location }\end{array}$ \\
\hline Abdessalam et al. ${ }^{6}$ & 0 & $\bullet^{\mathrm{a}}$ & - & - & 0 & 0 & 0 & 0 & 0 & 0 & & 0 & 0 & \\
\hline Weiser et al. ${ }^{7}$ & - & - & & - & 0 & 0 & & & 0 & & & & & 0 \\
\hline Rahusen et al. ${ }^{8 b}$ & 0 & - & & 0 & 0 & 0 & 0 & & & - & & & 0 & \\
\hline Wong et al. ${ }^{9}$ & - & & & & 0 & 0 & & & & - & 0 & & & 0 \\
\hline Turner et al. ${ }^{10 \mathrm{~b}}$ & $\bullet$ & $\bullet^{\mathrm{a}}$ & $\bullet$ & $\bullet$ & $\bullet$ & 0 & 0 & 0 & & $\bullet$ & & 0 & 0 & \\
\hline Hwang et al. ${ }^{11}$ & • & - & 0 & $\bullet$ & 0 & 0 & 0 & 0 & 0 & 0 & $\bullet$ & 0 & & \\
\hline Reynolds et al. ${ }^{12 b}$ & - & $\bullet$ & & 0 & 0 & 0 & $\bullet$ & 0 & 0 & & & & & \\
\hline Cserni $^{13}$ & $\bullet$ & $\bullet$ & $\bullet$ & & 0 & & & & & & & & & \\
\hline Kamath et al..$^{14}$ & $\bullet$ & $\bullet^{\mathrm{a}}$ & & & & & & & & & & & & \\
\hline Mignotte et al. ${ }^{15}$ & $\mathrm{O}^{\mathrm{c}}$ & $\mathrm{o}^{\mathrm{a}, \mathrm{c}}$ & & & & & & & & & & & & \\
\hline Canavese et al. ${ }^{16}$ & $o^{c}$ & $0^{c}$ & & & & & & & & & & & & \\
\hline
\end{tabular}

NSN: nonsentinel lymph nodes; LVI: lymphovascular invasion; ER: estrogen receptor; PR: progesterone receptor; SNs: sentinel lymph nodes; Open circles; feature evaluated but not found to be statistically significant by univariate analysis; Solid circles: feature found to be Statistically significant by univariate analysis.

${ }^{a}$ Included stratification for lymph nodes positive by immunohistochemistry only.

${ }^{\mathrm{b}}$ Statistical significance calculation based on data that included nonsentinel lymph nodes determined to be positive with routine use of immunohistochemistry.

c Statistical analysis not reported.

confined to the sinusoid of a SN was associated with a lower likelihood of positive NSNs than a metastasis in the parenchyma of the SN. In the second study, ${ }^{11}$ patients receiving neoadjuvant chemotherapy prior to axillary lymph node surgery were more likely to have positive NSNs. The third study found that utilizing IHC as the method of detection of metastasis in the SN was correlated with a lower likelihood of NSN metastasis, ${ }^{10}$ although it could be argued this is a surrogate assessment of size of metastasis in the SN. Other characteristics evaluated that were not found to be statistically significant included type of breast procedure, ${ }^{9}$ mitotic count ${ }^{12}$ or mitotic activity index, ${ }^{8}$ palpable axillary lymph node, ${ }^{10}$ S-phase fraction, ${ }^{10}$ and DNA ploidy. ${ }^{10}$

The apparent increase in the proportion of positive NSNs as the tumor size increases is shown in Figure 1. Despite some variability among the studies, there appears to be general concordance that the proportion of patients with positive NSNs increases with increasing tumor size. The largest variability in the proportions of patients with positive NSNs across studies was found to occur in the subgroups with the fewest patients (T1a, T3, and T4). The small subgroup sample sizes may have contributed to this variability.

A positive association between the size of the metastasis in the SN and the proportion of patients with positive NSNs is shown in Figure 2. In patients with micrometastatic $(\leq 2 \mathrm{~mm})$ SN disease, approximately $13-22 \%$ are reported to have additional metastasis by $\mathrm{H}$ \& $\mathrm{E}$ staining in NSNs, in contrast to

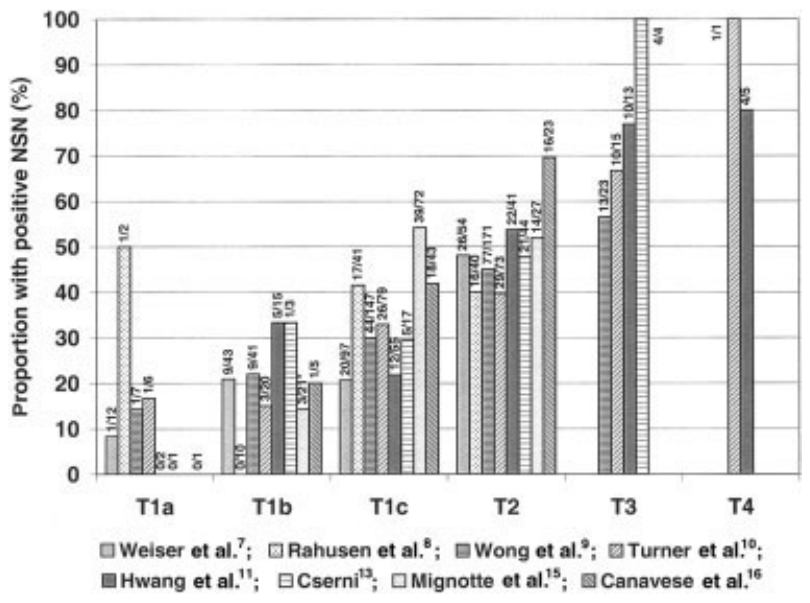

FIGURE 1. Effect of tumor size on the likelihood of positive nonsentinel lymph nodes (NSN). The numbers indicate the proportion of patients with positive NSN (by hematoxylin and eosin evaluation) for each subgroup. Please note that the data from the study by Rahusen et al. ${ }^{8}$ included positive NSNs that were detected with routine immunohistochemistry. The asterisk indicates that T1a and $\mathrm{T} 1 \mathrm{~b}$ patients were combined in this group.

$45-79 \%$ of patients with macrometastatic $(>2 \mathrm{~mm})$ disease. In those studies that classified the SN metastasis size as detected by IHC only, $\leq 2 \mathrm{~mm}$ excluding IHC-detected disease, or $>2 \mathrm{~mm}$, there was a trend toward further discrimination among patients with micrometastatic disease (Fig. 3). It is notable that even in the subgroup of patients with SN micrometastases that were detectable only with IHC, the chance of H \& E-positive NSNs was found to reach $20 \%$ (Fig. 3 ). 


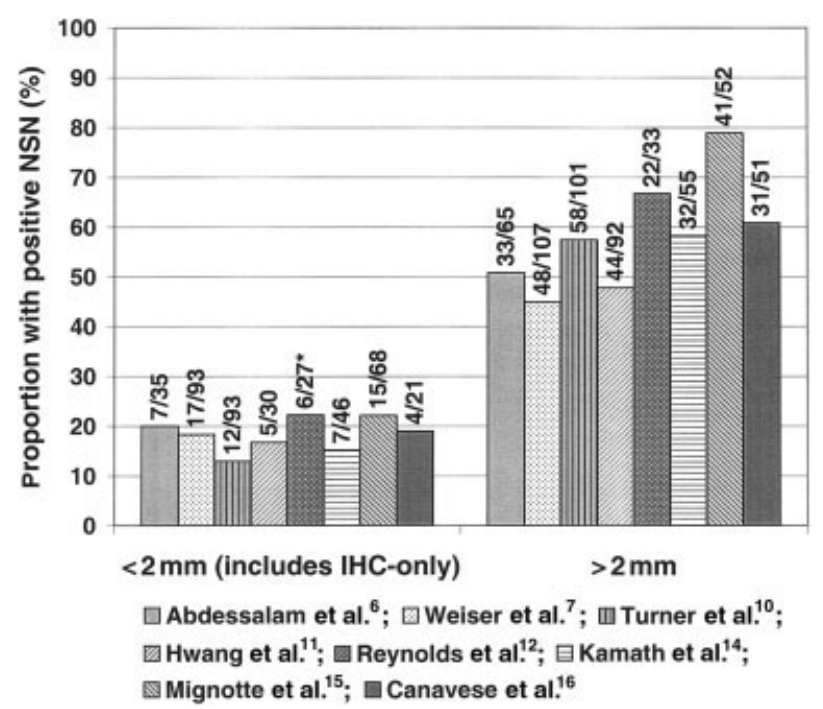

FIGURE 2. Effect of metastasis size on the likelihood of positive nonsentinel lymph nodes (NSN). The numbers indicate the proportion of patients with positive NSN (by hematoxylin and eosin evaluation) for each subgroup. The asterisk indicates that the data included positive NSNs detected with routine immunohistochemistry.

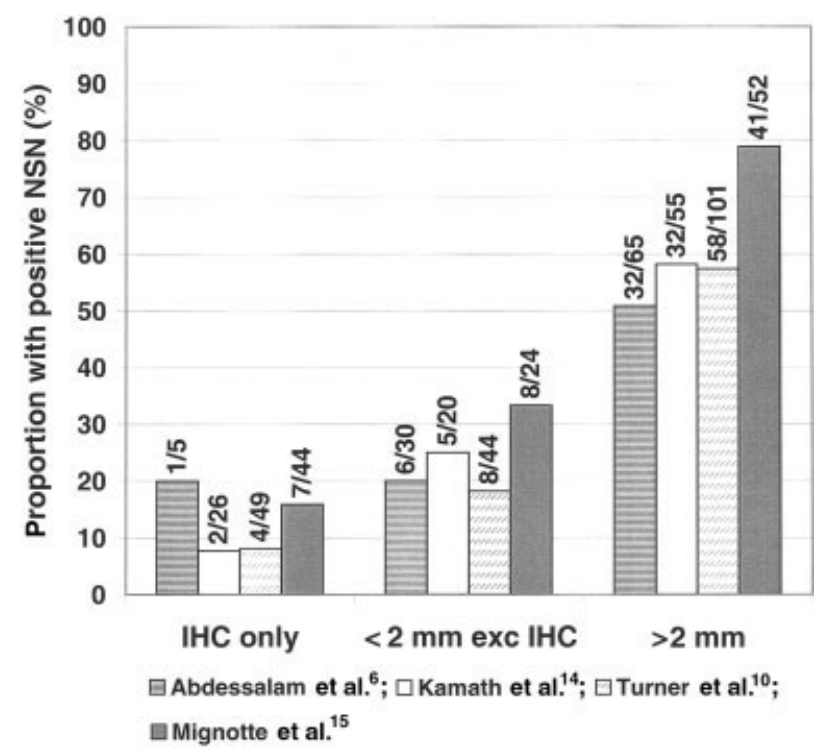

FIGURE 3. Effect of metastasis size (immunohistochemistry [IHC] only) on the likelihood of positive nonsentinel lymph nodes (NSN). The numbers indicate the proportion of patients with positive NSN (by hematoxylin and eosin evaluation) for each subgroup.

Three of four studies evaluating EE found a statistically higher proportion of patients with positive NSNs when EE was present (Fig. 4). Of the six studies that evaluated LVI, four found a statistically significant increase in the likelihood of positive NSNs when LVI was present. However, the magnitude of this differ-

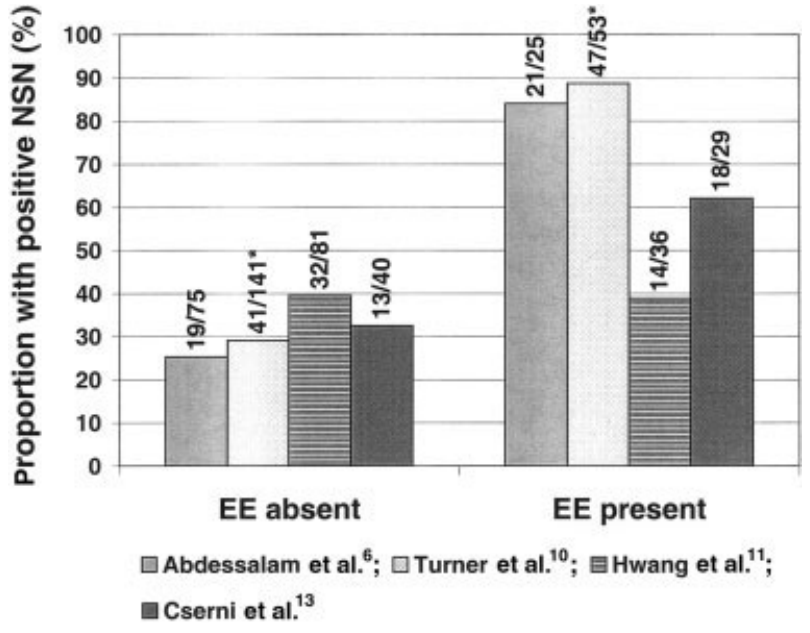

FIGURE 4. Effect of extranodal extension (EE) on the likelihood of positive nonsentinel lymph nodes (NSN). The numbers indicate the proportion of patients with positive NSN (by hematoxylin and eosin evaluation) for each subgroup. The asterisk indicates that the data included positive NSNs detected with routine immunohistochemistry $(\mathrm{IHC})$.

ence was less than that noted for tumor size, metastasis size, or EE.

The estimates of the common odds ratios for specified characteristics across studies, using the Mantel-Haenszel method, are shown in Figure 5. The common odds ratio and its $95 \% \mathrm{CI}$ are presented. Intervals that did not overlap the referent (1.0) were considered to be statistically significant at the $P<0.05$ level. Table 3 reports the total sample size and the sample distribution for the levels of the characteristics compared in Figure 5, along with the range of the calculated study-specific odds ratios. Using evaluation of the common odds ratios, the individual characteristics that demonstrated the strongest association with NSN metastasis were SN metastasis measuring $>2$ $\mathrm{mm}$, the presence of EE in the $\mathrm{SN}$, tumor size $>2 \mathrm{~cm}$, $>1$ positive $\mathrm{SN}$, and LVI present in the primary tumor. To our knowledge, these five characteristics also are the only ones reported to be significantly associated by the majority of studies that evaluated these factors (Table 2).

Multivariate analysis performed in several of the studies $^{7,9-13}$ also confirmed the importance of these five characteristics (Table 4). Each of the five characteristics with the strongest association by the MantelHaenszel meta-analysis was found to be statistically significant by multivariate analysis in at least one study, and there were no other features found to be significant by multivariate analysis. One study reported that metastasis size, LVI, and EE were all so highly related that on multivariate analysis only one 


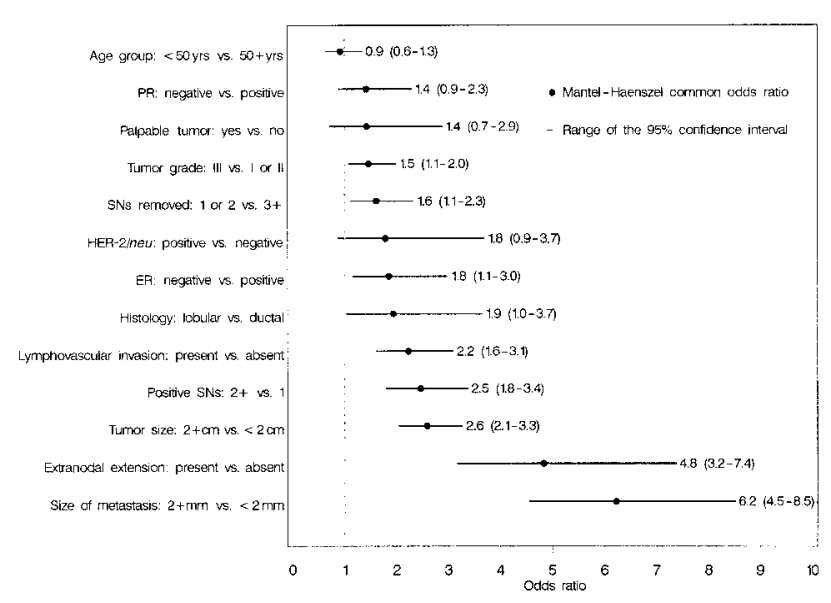

FIGURE 5. Mantel-Haenszel meta-analysis of odds ratios for selected features. PR: progesterone receptor; SNs: sentinel lymph nodes; ER: estrogen receptor.

factor remained significant, although this factor was not specified. ${ }^{6}$

\section{DISCUSSION}

Because of the lower morbidity associated with SN biopsy compared with ALND, ${ }^{23}$ this method has become widely utilized to stage the axilla in patients with breast carcinoma. The primary benefit offered by SN biopsy is the elimination of ALND in patients with lymph node-negative disease. When a $\mathrm{SN}$ is positive, completion ALND remains the standard of care. However, persistent concern for the morbidity of ALND generates a parallel question in SN-positive patients: can any of these individuals be spared a completion ALND? To consider omitting completion ALND, clinicians need information regarding the likelihood of additional axillary lymph node metastasis, as well as outcomes when potential residual disease is not resected.

The American College of Surgeons Oncology Group (ACOSOG) is currently accruing patients to a prospective trial that randomizes SN-positive lumpectomy patients to completion ALND versus observation. $^{24}$ We believe this study will provide definitive data regarding the necessity of a standard ALND in contributing to survival, as well as in obtaining durable regional control of disease after resection of at least one metastatic SN. Encouraging patients to participate in this important trial should be a high priority. However, it is likely that its mature results will not be reported for several years. In the meantime, a predictive model to estimate the likelihood of a patient harboring metastatic NSNs will remain valuable for those cases in which the ACOSOG trial is not available, and for patients who are ineligible for the study, such as mastectomy patients. Knowledge of the clinicopathologic characteristics associated with NSN metastasis may be helpful in the analysis of data and correlation with outcome in this trial or future investigations.

The results of the current meta-analysis demonstrate that in the presence of any 1 of 5 characteristics (SN metastasis size $>2 \mathrm{~mm}$, the presence of extranodal extension in the $\mathrm{SN}$, tumor size $>2 \mathrm{~cm},>1$ positive SN, or LVI present in the primary tumor), there is a $>2$-fold increase in the likelihood of finding additional metastasis in NSNs. Conversely, no subgroup of patients identified by a single characteristic consistently demonstrated a $<10 \%$ chance of NSN metastasis. Theoretically, patients lacking all five of the pathologic features characteristics specified would comprise this group; however, effective analysis for this narrowly defined subgroup is limited by small patient numbers, underscoring the importance of a large dataset.

Although the data presented in the current study are limited to the strength of associations of the individual characteristics, there is remarkable similarity reported among studies despite known differences in protocols. One of the known differences-the variable use of IHC to evaluate NSNs-is a concerning potential source of variation among the studies. As mentioned previously, in patients with $\mathrm{H}$ \& E- or IHCpositive SNs, increased scrutiny of NSNs with IHC and additional sections is reported to result in a $10-20 \%$ increase in the detection of NSN metastasis. ${ }^{21,22}$ Five studies included in the meta-analysis reported the routine use of IHC, three did not routinely use IHC, and in three studies the use of IHC was indeterminate. Including studies that varied with regard to the use of IHC on NSNs appears to decrease the homogeneity of the dataset in the meta-analysis, but excluding studies on this basis would markedly limit the size of the dataset. In our opinion, the larger dataset afforded by including all the studies justified this potential source of variability. Furthermore, the graphical representation of study-specific results in Figures 1-4 allows the reader to correlate results based on the likelihood of positive NSNs with use of IHC.

Based on the meta-analysis, the size of the metastatic focus in the $\mathrm{SN}$ appears to demonstrate the strongest association with the likelihood of positive NSNs, and the lowest likelihood of positive NSNs noted for any single characteristic occurred in the subgroup with IHC-detected metastasis. Although controversial, the use of IHC on SNs is not recommended currently. ${ }^{25}$ Therefore, a predictive model that incorporates this feature may have compromised 
TABLE 3

Total Sample Size and Range of Study-Specific Univariate ORs

\begin{tabular}{|c|c|c|c|c|c|}
\hline \multirow[b]{2}{*}{ Covariate } & \multirow[b]{2}{*}{ Level A (\%) } & \multirow[b]{2}{*}{ Level B (\%) } & \multirow[b]{2}{*}{ Sample size } & \multicolumn{2}{|c|}{$\begin{array}{l}\text { Range of study-specific ORs, } \\
\text { Comparing Level A with Level B }\end{array}$} \\
\hline & & & & Minimum & Maximum \\
\hline Age group (yrs) & $<50(49)$ & $50+(51)$ & 467 & 0.63 & 1.05 \\
\hline Progesterone receptor & Negative (38) & Positive (62) & 290 & 1.16 & 1.94 \\
\hline Palpable tumor & Yes (72) & No (28) & 170 & 0.73 & 2.00 \\
\hline Tumor grade & III (39) & I or II (61) & 789 & 0.69 & 2.38 \\
\hline SNs removed & 1 or $2(62)$ & $3(38)$ & 520 & 1.31 & 2.93 \\
\hline HER-2/neu & Positive (25) & Negative (75) & 165 & 1.40 & 2.13 \\
\hline Estrogen receptor & Negative (27) & Positive (73) & 354 & 1.23 & 3.57 \\
\hline Histology & Lobular (9) & Ductal (91) & 481 & 1.45 & 5.03 \\
\hline Lymphovascular invasion & Present (33) & Absent (67) & 719 & 0.60 & 3.62 \\
\hline No. of positive SNs & $2+(29)$ & $1(71)$ & 807 & 1.98 & 5.40 \\
\hline Tumor size (cm) & $2+(42)$ & $<2(58)$ & 1,334 & 1.29 & 11.40 \\
\hline Extranodal extension & Present (30) & Absent (70) & 480 & 0.97 & 15.47 \\
\hline Size of metastasis (mm) & $2+(57)$ & $<2(43)$ & 969 & 3.64 & 13.17 \\
\hline
\end{tabular}

ORs: odds ratios; SN: sentinel lymph nodes.

TABLE 4

Significance of Selected Features by Multivariate Analysis

\begin{tabular}{llllll}
\hline Study & $\begin{array}{l}\text { Tumor } \\
\text { size }\end{array}$ & $\begin{array}{l}\text { Metastasis } \\
\text { size }\end{array}$ & EE & LVI & $\begin{array}{l}>\text { SN positive } \\
\text { SN }\end{array}$ \\
\hline Weiser et al. $^{7}$ & $\bullet$ & $\bullet$ & & 0 & \\
Wong et al. $^{9}$ & $\bullet$ & & & & $\bullet$ \\
Turner et al. $^{10}$ & $\bullet$ & $\bullet$ & $\bullet$ & $\bullet$ & 0 \\
Hwang et al. $^{11}$ & $\bullet$ & $\bullet$ & 0 & $\bullet$ & 0 \\
Reynolds et al. $^{12}$ & $\bullet$ & $\bullet$ & & 0 & \\
Cserni $^{13}$ & $\bullet$ & $\bullet$ & $\bullet$ & & \\
\hline
\end{tabular}

EE: extranodal extension; LVI: lymphovascular invasion; SN: sentinel lymph node; Solid circle: feature found to be statistically significant on multivariate analysis; Open circle: feature evaluated, but not found to be statistically significant by multivariate analysis.

applicability when pathologic evaluation of the SN does not include IHC. IHC detection of metastasis in the SN is essentially a surrogate determination of small metastasis size. Because the size of the metastasis in the SN appears to be the single most important feature, a predictive model based on a more refined categoric distinction of metastasis size (or incorporation of the actual measured size of the metastasis as a continuous variable) might provide an alternative and more applicable approach to distinguish those patients least likely to have NSN metastasis. This potential advantage would have to be weighed against the disadvantage of requiring a pathologic measurement that is not routinely reported.

Rahusen et al. ${ }^{8}$ suggested that redefining micrometastasis as lymph node disease $<1 \mathrm{~mm}$ may provide better discrimination of patients at low risk for metastasis in NSNs. Support for this idea is found in a study that focused exclusively on patients with SN metastasis $\leq 2 \mathrm{~mm}$, subdividing patients by metastasis size in $0.3-\mathrm{mm}$ increments. ${ }^{18}$ The authors found that the proportion of patients with positive NSNs was $13-17 \%$ for subgroups in which the metastasis was $\leq 1$ mm compared with $33-38 \%$ for subgroups with in which the metastasis measured1-2 mm. Similarly, Rahusen et al. found a difference in NSN metastasis using a $1-\mathrm{mm}$ cutoff for metastasis size $27 \%$ with SN metastasis $<1 \mathrm{~mm}$ compared with $50 \%$ with SN metastasis $\geq 1 \mathrm{~mm}$ ). ${ }^{8}$ These values appear high and may be explained by the use of routine IHC on NSNs, but they also support consideration of a $1-\mathrm{mm}$ cutoff. Although current American Joint Committee on Cancer criteria define micrometastatic disease as disease measuring $\leq 2 \mathrm{~mm}$, Rahusen et al. ${ }^{8}$ proposed that a 1 -mm delineation may be more clinically relevant with a goal of discriminating which individuals have a dramatically lower risk of NSN metastases and could potentially forego completion ALND.

Despite the findings on univariate analysis, confounding of variables is expected, and multivariate analysis is necessary to develop a model that could accurately predict the likelihood of positive NSNs in an individual patient. Unfortunately, without the original multivariate data regarding each individual person/patient in each study, we do not believe it is possible to perform multivariate analysis on the pooled data for this review. However, a multivariate model to predict the status of NSNs recently was proposed in an abstract from the Memorial Sloan-Ketter- 
ing Cancer Center based on a dataset of 702 patients with positive SNs who underwent completion ALND. ${ }^{26}$ A nomogram was presented that included nine factors, with points assigned for each factor. The researchers reported a predictive accuracy of $74 \%$ with this model. EE was not found to be one of the features included in their model, although it was statistically significant by univariate analysis in three studies ${ }^{6,10,13}$ and by multivariate analysis in one study reviewed herein. ${ }^{10}$ We believe the publication of this predictive model will afford the opportunity to validate it in a dataset from another institution.

If a low probability of NSN metastasis can be predicted, a judgment regarding the omission of completion ALND will remain subject to the opinions of both physicians and patients. Individuals advocating elimination of ALND whenever possible will argue that it may not be necessary to resect residual disease. The multiple articles reviewed in the current study demonstrate that only $20-30 \%$ of patients with micrometastatic disease will have metastatic disease in the NSNs. Some of these patients would receive cytotoxic chemotherapy or radiation therapy (regardless of the NSN status), which might successfully eradicate any residual axillary lymph node disease. Guenther et al. ${ }^{27}$ reported a low incidence of regional failure in 46 patients with SN metastases who did not undergo ALND (mean follow-up of 32 months). Furthermore, Greco et $\mathrm{al}^{28}$ documented that axillary lymph node recurrences in untreated axillas are less frequent than would be expected based on the incidence of positive lymph nodes with ALND, implying that only a small proportion of patients with histologically detectable axillary lymph node disease will develop a clinical recurrence.

In contrast, individuals who support more aggressive surgical management will point out that ALND improves local disease control and also may provide a survival benefit. A review of survival data from the National Cancer Database concerning women with Stage I and Stage II breast carcinoma demonstrated that the 10 -year survival was significantly worse when ALND was omitted, and this effect was not completely the result of understaging. ${ }^{29}$ However, to our knowledge, data regarding comorbidities that may have influenced the decision to minimize the aggressiveness of surgical care were not available. Further support for ALND is found in a meta-analysis of randomized controlled trials that demonstrated a $5 \%$ survival benefit to ALND, although these patients did not receive adjuvant therapy, limiting the applicability of these data to current practice. ${ }^{30}$ Another factor that supports completion ALND is the finding of macroscopic residual disease in some patients whose SNs have only micrometastatic disease; among those studies reporting this finding, the incidence rate was reported to be as high as $16 \% .{ }^{8,10,17,18}$ Although it may appear logical to eliminate completion ALND for patients unlikely to have positive NSNs, Morrow argues that patients with small primary tumors and only one to three positive lymph nodes may be those individuals most likely to obtain a survival benefit from aggressive locoregional therapy. ${ }^{31}$

With the therapeutic value of ALND still in question, information regarding the likelihood of metastasis in NSNs can be very helpful when counseling breast carcinoma patients with positive SNs. The articles reviewed in the current study underscore the fact that this is a common issue in clinical practice. Until results are available from the ACOSOG Z0011 trial regarding the therapeutic value of ALND, a predictive model will be valuable to clinicians. The analysis presented herein suggests that the five characteristics most strongly associated with NSN metastasis should be included in prospective databases and in the formulation of a predictive model. The development and validation of this model will require multivariate analysis of a large dataset from different institutions. Efforts currently are underway to achieve this goal. Given the similarity of the data from different institutions in the current review, it is reasonable to be optimistic concerning the widespread applicability of a validated predictive model. The use of any predictive model will nonetheless require clinical judgment weighing the risks and benefits of completion ALND.

\section{REFERENCES}

1. Petrek JA, Senie RT, Peters M, Rosen PP. Lymphedema in a cohort of breast carcinoma survivors 20 years after diagnosis. Cancer. 2001;92:1368-1377.

2. Kakuda JT, Stuntz M, Trivedi V, Klein SR, Vargas HI. Objective assessment of axillary morbidity in breast cancer treatment. Am Surg. 1999;65:995-998.

3. Chu KU, Turner RR, Hansen NM, Brennan MB, Bilchik A, Giuliano AE. Do all patients with sentinel node metastasis from breast carcinoma need complete axillary node dissection? Ann Surg. 1999;229:536-541.

4. Liang WC, Sickle-Santanello BJ, Nims TA. Is a completion axillary dissection indicated for micrometastases in the sentinel lymph node? Am J Surg. 2001;182:365-368.

5. Mantel N, Haenszel W. Statistical aspects of the analysis of data from retrospective studies of disease. J Natl Cancer Inst. 1959;22:719-748.

6. Abdessalam SF, Zervos EE, Prasad M, et al. Predictors of positive axillary lymph nodes after sentinel lymph node biopsy in breast cancer. Am J Surg. 2001;182:316-320.

7. Weiser MR, Montgomery LL, Tan LK, et al. Lymphovascular invasion enhances the prediction of non-sentinel node metastases in breast cancer patients with positive sentinel nodes. Ann Surg Oncol. 2001;8:145-149. 
8. Rahusen FD, Torrenga H, van Diest PJ, et al. Predictive factors for metastatic involvement of nonsentinel nodes in patients with breast cancer. Arch Surg. 2001;136:10591063.

9. Wong SL, Edwards MJ, Chao C, et al. Predicting the status of the nonsentinel axillary nodes: a multicenter study. Arch Surg. 2001;136:563-568.

10. Turner RR, Chu KU, Qi K, et al. Pathologic features associated with nonsentinel lymph node metastases in patients with metastatic breast carcinoma in a sentinel lymph node. Cancer. 2000;89:574-581.

11. Hwang RF, Krishnamurthy S, Hunt K, et al. Clinicopathologic factors predicting involvement of nonsentinel axillary nodes in women with breast cancer. Ann Surg Oncol. 2003; 10:248-254.

12. Reynolds C, Donohue JH, Grant CS, et al. Sentinel lymph node biopsy with metastasis: can axillary dissection be avoided in some patients with breast cancer? J Clin Oncol. 1999;17:1720-1726.

13. Cserni G. Sentinel lymph-node biopsy-based prediction of further breast cancer metastases in the axilla. Eur J Surg Oncol. 2001;27:532-538.

14. Kamath VJ, Giuliano R, Dauway EL, et al. Characteristics of the sentinel lymph node in breast cancer predict further involvement of higher-echelon nodes in the axilla. Arch Surg. 2001;136:688-692.

15. Mignotte H, Treilleux I, Faure C, Nessah K, Bremond A. Axillary lymph-node dissection for positive sentinel nodes in breast cancer patients. Eur J Surg Oncol. 2002;28:623626

16. Canavese G, Gipponi M, Catturich A, et al. Technical issues and pathologic implications of sentinel lymph node biopsy in early-stage breast cancer patients. J Surg Oncol. 2001;77: 81-87.

17. Den Bakker MA, van Weeszenberg A, de Kanter AY, et al. Non-sentinel lymph node involvement in patients with breast cancer and sentinel node micrometastasis; too early to abandon axillary clearance. J Clin Pathol. 2002;55:932935.

18. Viale G, Maiorano E, Mazzarol G, et al. Histologic detection and clinical implications of micrometastases in axillary sentinel lymph nodes for patients with breast carcinoma. Cancer. 2001;92:1378-1384.

19. Sachdev U, Murphy K, Derzie A, Jaffer S, Bleiweiss IJ, Brower S. Predictors of nonsentinel lymph node metastasis in breast cancer patients. Am J Surg. 2002;183:213-217.
20. Turner RR, Ollila DW, Krasne DL, Giuliano AE. Histopathologic validation of the sentinel lymph node hypothesis for breast carcinoma. Ann Surg. 1997;226:271-278.

21. Sabel MS, Zhang P, Barnwell JM, Winston JS, Hurd T, Edge SB. Accuracy of sentinel node biopsy in predicting nodal status in patients with breast carcinoma. J Surg Oncol 2001; $77: 243-246$.

22. Chu KU, Turner RR, Hansen NM, Brennan MB, Giuliano AE Sentinel node metastasis in patients with breast carcinoma accurately predicts immunohistochemically detectable nonsentinel node metastasis. Ann Surg Oncol. 1999;6:756761.

23. Sener SF, Winchester DJ, Martz CH, et al. Lymphedema after sentinel lymphadenectomy for breast carcinoma. Cancer. 2001;92:748-752.

24. Grube BJ, Giuliano AE. Observation of the breast cancer patient with a tumor-positive sentinel node: implications of the ACOSOG Z0011 trial. Semin Surg Oncol 2001;20: 230-237.

25. Schwartz GF, Giuliano AE, Veronesi U. Proceedings of the consensus conference on the role of sentinel lymph node biopsy in carcinoma of the breast, April 19-22, 2001, Philadelphia, Pennsylvania. Cancer. 2002;94:2542-2551.

26. Bevilacqua JL, Manasseh DE, Kattan MW, et al. A multivariate nomogram to predict likelihood of non-sentinel node metastases in breast cancer patients with positive sentinel nodes [abstract]. Presented at the Society of Surgical Oncology, Los Angeles, California, March 6-9, 2003.

27. Guenther JM, Hansen NM, DiFronzo LA, et al. Axillary dissection is not required for all patients with breast cancer and positive sentinel nodes. Arch Surg. 2003;138: $52-56$.

28. Greco M, Agresti R, Cascinelli N, et al. Breast cancer patients treated without axillary surgery: clinical implications and biologic analysis. Ann Surg. 2000;232:1-7.

29. Bland KI, Scott-Conner CE, Menck H, Winchester DP. Axillary dissection in breast-conserving surgery for Stage I and II breast cancer: a National Cancer Data Base study of patterns of omission and implications for survival. J Am Coll Surg. 1999;188:586-596.

30. Orr RK. The impact of prophylactic axillary node dissection on breast cancer survival-a Bayesian meta-analysis. Ann Surg Oncol. 1999;6:109-116.

31. Morrow M. A survival benefit from axillary dissection: was Halsted correct? Ann Surg Oncol. 1999;6:17-18. 